\title{
Determinação da idade fetal por meio da técnica ultra-sonográfica de fetometria e de morfologia fetal em cabras
}

\author{
[Determination of fetal age in goats by real-time ultrasound fetometry and fetal morphology] \\ E. Léga ${ }^{1}$, G.H. Toniollo ${ }^{2 *}$, J.A. Oliveira ${ }^{2}$, K.T. Resende ${ }^{2}$, V. Rodrigues ${ }^{2}$ \\ ${ }^{1}$ Curso de Medicina Veterinária do Centro Universitário Moura Lacerda \\ ${ }^{2}$ Faculdade de Ciências Agrárias e Veterinárias - UNESP \\ Via de Acesso Prof. Paulo Donato Castellane, s/n \\ 14884-900 - Jaboticabal, SP
}

\begin{abstract}
RESUMO
Utilizaram-se 13 cabras gestantes da raça Saanen, para estudo das características da gestação a partir do $60^{\circ}$ dia, segundo as técnicas de fetometria e avaliação morfológica fetal, utilizando-se ultra-sonografia em modo-B. Analisaram-se: diâmetro orbital (DO), diâmetro interorbital (DIO), diâmetro biorbital (DBO), diâmetro biparietal (DBP), comprimento do fêmur (CF), comprimento da tíbia (CT), comprimento do rádio (CR), diâmetro do tórax (DT), diâmetro abdominal transversal (DAT), diâmetro abdominal anteroposterior (DAP), diâmetro occípito-frontal (DOF), diâmetro transversal renal (RIMT), diâmetro longitudinal renal (RIML), comprimento da escápula (ESC), comprimento do metacarpo (MEC), medida da pelve (COX) e comprimento do úmero (CU). As médias dos valores achados foram correlacionadas com a idade fetal. As equações de regressão inversa foram criadas para cada parâmetro, sendo que DBP $\left(\mathrm{R}^{2}=0,93\right), \mathrm{CF}\left(\mathrm{R}^{2}=0,95\right), \mathrm{CU}\left(\mathrm{R}^{2}=0,96\right)$, $\mathrm{DO}\left(\mathrm{R}^{2}=0,84\right)$ e DAT $\left(\mathrm{R}^{2}=0,91\right)$ foram as variáveis de melhor correlação estatística, cuja equação pode ser representada pela fórmula: idade fetal $=34,5+4,8 \mathrm{DBP}+9,9$ $\mathrm{CF}-7,5 \mathrm{CU}-2,3 \mathrm{DO}+6,1 \mathrm{DAT}$.
\end{abstract}

Palavras-chave: cabra, ultra-sonografia, gestação, fetometria

\begin{abstract}
Fetal morphology analysis and real-time ultrasound techniques were used on 13 pregnant Saanen goats for studying the gestational characteristics. Analysis began on the $60^{\text {th }}$ day of pregnancy and the parameters observed were: orbital diameter (OD), inter-orbiall diameter (IOD), bi-orbital diameter $(B O D)$, bi-parietal diameter (BPD), femoral length ( $F L)$, tibial length (LT), radius length (RL), thoracic diameter (TD), transversal abdominal diameter (TAD), anteroposterior abdominal diameter (APAD), occipital-frontal diameter (OFD), transversal renal diameter (TRD), longitudinal renal diameter (LRD), scapular length (SL), metacarpal length $(M L)$, pelvic length $(P L)$ and humeral length $(H L)$. Mean values were correlated to fetal age and inverse regression equations were created for each parameter. Results obtained showed BPD ( $\left.R^{2}=0.93\right), F L\left(R^{2}=0.95\right), H L\left(R^{2}=0.96\right), O D\left(R^{2}=0.84\right)$ and $T A D\left(R^{2}=0.91\right)$ as the parameters with better statistical correlation to fetal age using the equation: Fetal age $=34.5+$ 4.8BPD + 9.9 FL - 7.5 HL - 2.3 OD + 6.1 TAD.
\end{abstract}

Keywords: goats, ultrasound, pregnancy, fetometry

Recebido em 5 de maio de 2006

Aceito em 4 de abril de 2007

*Autor para correspondência (corresponding author)

E-mail: toniollo@fcav.unesp.br 


\section{INTRODUÇÃO}

O estudo da gestação, desde sua detecção precoce até a determinação da data de parto tem como principais benefícios: (a) a programação e a diferenciação do rebanho, (b) o diagnóstico de problemas relacionados à fertilidade ou a defeitos congênitos, (c) o descarte precoce de fêmeas inférteis e (d) a suplementação alimentar de fêmeas gestantes, principalmente no terço final da gestação (Simplício, 2000; Salles, 2001).

Frente ao avanço na utilização de programas de indução e/ou sincronização de cios, desenvolvimento de métodos de inseminação artificial e transferência de embriões, faz-se necessária a aplicação de método eficiente de diagnóstico de gestação e de estimativa da idade fetal (Simplício, 2000; Salles, 2001). Associamse a essa observação os relatos de Al-Marestani et al. (2003), em que a produtividade dos rebanhos não está relacionada somente ao número de fêmeas gestantes num período, mas também ao número de filhotes produzidos por ano.

A ultra-sonografia em modo-B tem superado os vários métodos utilizados para o diagnóstico de gestação em cabras por ter elevada acurácia, não apresentar efeitos deletérios para a fêmea, feto ou operador, não ser invasiva, ser método rápido, prático e satisfatório no diagnóstico de gestação, determinação do número de fetos e realização da fetometria e avaliação da morfologia fetal para determinação da fase de desenvolvimento de um feto in vivo (Chavez Moreno et al., 1996; Léga, 2000), visto que várias dimensões lineares podem ser correlacionadas com a idade fetal (Reichle e Haibel, 1991; Ishwar, 1995).

Embora alguns autores considerem a estimativa da idade fetal mera especulação (Trigo, 2002), o estudo ultra-sonográfico dos aspectos morfológicos pode ser correlacionado com alterações causadas mediante tratamentos dietéticos especiais (Kusina et al., 2001), farmacológicos (Panter et al., 2000) ou de manejo (Preez et al., 2001). Assim, acompanhando a evolução biológica, a ultrasonografia também tem dado respaldo para estabelecer o sucesso na geração de clones em receptoras pela avaliação morfológica destes produtos durante a gestação (Keefer et al., 2002; Zou, 2002).
A fetometria mostra ser apropriada para determinação da idade fetal, sendo que até o $42^{\circ}$ dia de gestação, os parâmetros mais úteis são o comprimento craniocaudal e o diâmetro médio da vesícula gestacional (Léga, 2000). A partir do momento da calcificação fetal, o comprimento de ossos longos pela mensuração de suas diáfises, medidas oculares incluindo diâmetros interorbital, orbital e biorbital, medidas do crânio, incluindo diâmetros biparietal e occípitofrontal, diâmetros do tórax e abdominais láterolateral e ventrodorsal apresentam alta acurácia (Sauerbrei et al., 1992; Bailão, 1998; Chalhoub et al., 1998).

Souza et al. (2002) demonstraram que as variáveis independentes diâmetro biparietal e diâmetro abdominal tiveram alta significância para a determinação da idade fetal, visto que apresentaram maiores valores de coeficientes de determinação $(0,96)$.

Messias et al. (2001) referiram que pode haver erros na análise fetométrica feita pela ultrasonografia, especialmente em fetos maiores, necessitando-se obter o maior número de mensurações possível.

Assim, o objetivo deste estudo foi realizar exames ultra-sonográficos sucessivos para a observação da fetometria, avaliação do perfil biofísico do feto, que inclui a observação dos seus movimentos cardíacos e somáticos, e realização de exame morfológico a partir do $60^{\circ}$ dia de gestação, na espécie caprina, para determinar a idade fetal.

\section{MATERIAL E MÉTODOS}

Entre os meses de abril a outubro de 2001, em um rebanho, foram observadas 25 cabras da raça Saanen, com idades entre 10 e 48 meses, e que apresentavam atividade sexual cíclica normal, das quais 17 foram vistas acasalando com macho de fertilidade comprovada.

Após 40 dias do acasalamento, as cabras foram submetidas a exame ultra-sonográfico para diagnóstico de gestação e determinação do número de fetos, sendo 13 selecionadas para estudo ultra-sonográfico fetal a partir do $60^{\circ}$ dia após o acasalamento, até o dia do parto. 
A avaliação ultra-sonográfica foi realizada em modo-B empregando-se aparelho Pie-Medical, mod. Scanner $200 \mathrm{Vet}$, com transdutor mecânico setorial de 5,0 $\mathrm{MHz}$ e/ou transdutor linear eletrônico de $5,0 \mathrm{mHz}$, posicionado em região inguinal previamente tricotomizada com máquina de tosa Oster, modelo $\mathrm{A} 5$, de duas velocidades e lâmina 40 e em seguida lubrificada com gel comercial, mantendo-se o animal em posição quadrupedal.

Os exames morfológico e fetométrico foram realizados a partir do $60^{\circ}$ dia após o acasalamento, seguindo-se de acompanhamento com freqüência de duas a três vezes por semana, com intervalos máximos de três dias entre uma observação e outra, até o dia do parto, quando a determinação do número fetal e a eficiência do diagnóstico puderam ser confirmadas.

As imagens foram registradas conforme proposto por Léga (2000), sendo mensuradas as seguintes variáveis: diâmetro interorbital (DIO), comprimento craniocaudal (CCC), diâmetro biparietal (DBP), freqüência cardíaca (FC), comprimento do fêmur $(\mathrm{CF})$, comprimento da tíbia (CT), comprimento do rádio (CR), comprimento do úmero (CU), diâmetro orbital (DO), diâmetro biorbital (DBO), diâmetro do tórax (DT), diâmetro abdominal transversal (DAT), diâmetro abdominal ânteroposterior ou ventrodorsal (DAP), diâmetro occípito-frontal (DOF), diâmetro renal transversal (RIMT), diâmetro renal longitudinal (RIML), comprimento da escápula (ESC), comprimento do metacarpo (MEC), comprimento do metatarso (MET), comprimento da pelve (COX), circunferência craniana (CC), comprimento da vértebra lombar (VL), circunferência abdominal (CA), diâmetro umbilical (DU) e diâmetro da artéria aorta (DAORT).

Em todos os animais procurou-se realizar o maior número de medidas possível, examinandose o abdômen nos dois antímeros. As médias dos dados obtidos foram organizadas em forma de tabela, por idade fetal, considerando-se o número de dias após o acasalamento e cada variável.

A relação entre a média das variáveis fetométricas FC, DIO, DBP, CT, CR, DO, DBO, DT, CF, CU, DAT, DAP, DOF, RIMT, RIML, ESC, MEC e COX e a idade gestacional foi estudada por meio de análise de regressão linear simples (Montgomery e Peck, 1982; Diggle et al., 1995). Todas as variáveis analisadas foram consideradas dependentes do estádio gestacional.

As variáveis $\mathrm{FC}, \mathrm{DBP}, \mathrm{CF}, \mathrm{CU}, \mathrm{DO}, \mathrm{DT}$ e DAT foram submetidas à análise de regressão linear múltipla, sendo que a seleção dos modelos foi realizada pelo método backward, objetivando criar uma equação com modelo reduzido.

Para predição da idade gestacional a partir das medidas fetais, realizou-se regressão inversa do modelo de regressão linear ajustado em cada caso (Montgomery e Peck, 1982), sendo que, para a regressão linear $(\mathrm{y}=\mathrm{a}+\mathrm{bx})$, a regressão inversa foi dada por:

$$
\mathrm{x}=(\mathrm{y}-\mathrm{a}) / \mathrm{b} \text {, em que: }
$$

$\mathrm{x}=$ variável independente que é a idade gestacional em dias;

$$
\mathrm{a}=\text { intercepto; }
$$

$\mathrm{b}=$ efeito linear da variável independente (inclinação da reta);

$\mathrm{y}=$ variável mensurada (medidas embrionárias/fetais)

Para as análises estatísticas das características avaliadas foi utilizado o programa SAS (User's..., 1996).

\section{RESULTADOS}

Várias estruturas puderam ser mensuradas a partir das imagens ultra-sonográficas, sendo, então, utilizada a fetometria em secções horizontais, longitudinais ou sagitais e transversais, para se criar uma correlação entre as estruturas mensuradas e a idade fetal a partir da média de cada variável calculada por período. Os parâmetros que apresentaram menor número de observações não foram utilizados para a análise estatística, visto que os posicionamentos adotados pelos fetos durante os exames influenciaram diretamente a obtenção das imagens.

A duração média da gestação foi de 150,85 dias, com desvio-padrão de 1,77. A partir dos dados fetométricos obtidos, pôde-se criar uma relação entre as estruturas fetais, determinando-se, assim, suas equações de regressão inversa para determinação das idades fetais (Tab. 1). 
Tabela 1. Equações das regressões lineares, coeficientes de determinação $\left(\mathrm{R}^{2}\right)$, probabilidade de significância e equações da regressão linear inversa das características usadas nos exames de ultrasonografia obstétrica transabdominal em cabras Saanen

\begin{tabular}{|c|c|c|c|}
\hline $\begin{array}{c}\text { Parâmetro fetométrico } \\
\text { e regressão linear }\end{array}$ & $\mathrm{R}^{2}$ & Significância & Regressão inversa \\
\hline$\overline{\mathrm{DIO}}=-0,09+0,01$ dias & 0,67 & $\mathrm{P}<0,01$ & Dias $=9,00+100,00$ DIO \\
\hline $\mathrm{DO}=-0,12+0,02$ dias & 0,84 & $\mathrm{P}<0,01$ & Dias $=6,00+50,00 \mathrm{DO}$ \\
\hline $\mathrm{DBO}=0,02+0,05$ dias & 0,89 & $\mathbf{P}<0,01$ & Dias $=-0,60+20,00 \mathrm{DBO}$ \\
\hline $\mathrm{DBP}=-0,55+0,04$ dias & 0,93 & $\mathrm{P}<0,01$ & Dias $=13,75+25,00$ DBP \\
\hline $\mathrm{DOF}=-1,32+0,06$ dias & 0,93 & $\mathbf{P}<0,01$ & Dias $=22,00+16,67 \mathrm{DOF}$ \\
\hline $\mathrm{CU}=-2,96+0,06 \mathrm{dias}$ & 0,96 & $\mathrm{P}<0,01$ & Dias $=49,33+16,67 \mathrm{CU}$ \\
\hline $\mathrm{CT}=-2,53+0,06$ dias & 0,95 & $\mathbf{P}<0,01$ & Dias $=42,17+16,67 \mathrm{CT}$ \\
\hline $\mathrm{CR}=-2,56+0,06$ dias & 0,93 & $\mathrm{P}<0,01$ & Dias $=42,67+16,67 \mathrm{CR}$ \\
\hline $\mathrm{CF}=-3,43+0.07$ dias & 0,95 & $\mathrm{P}<0,01$ & Dias $=49,00+14,29 \mathrm{CF}$ \\
\hline $\mathrm{DT}=-2,15+0,07$ dias & 0,95 & $\mathbf{P}<0,01$ & Dias $=30,71+14,29 \mathrm{DT}$ \\
\hline $\mathrm{DAT}=-2,37+0,08$ dias & 0,91 & $\mathrm{P}<0,01$ & Dias $=29,62+12,50$ DAT \\
\hline $\mathrm{DAP}=-1,51+0,07$ dias & 0,91 & $\mathbf{P}<0,01$ & Dias $=21,57+14,29$ DAP \\
\hline RIMT $=-0,04+0,02$ dias & 0,76 & $\mathbf{P}<0,01$ & Dias $=2,00+50,00$ RIMT \\
\hline RIML $=-0,03+0,02$ dias & 0,81 & $\mathbf{P}<0,01$ & Dias $=1,50+50,00$ RIML \\
\hline $\mathrm{ESC}=-3,00+0,06$ dias & 0,80 & $\mathbf{P}<0,01$ & Dias $=50,00+16,67 \mathrm{ESC}$ \\
\hline $\mathrm{MEC}=-2,66+0,05$ dias & 0,87 & $\mathrm{P}<0,01$ & Dias $=53,20+20,00 \mathrm{MEC}$ \\
\hline $\mathrm{COX}=-3,97+0,08$ dias & 0,88 & $\mathrm{P}<0,05$ & Dias $=49,62+12,50 \mathrm{COX}$ \\
\hline
\end{tabular}

DIO: diâmetro interorbital; DO: diâmetro orbital; DBO: diâmetro biorbital; DBP: diâmetro biparietal; DOF: diâmetro occípito-frontal; CU: comprimento do úmero; CT: comprimento da tíbia; CR: comprimento do rádio; CF: comprimento do fêmur; DT: diâmetro do tórax; DAT: diâmetro abdominal transversal; DAP: diâmetro abdominal ânteroposterior ou ventrodorsal; RIMT: diâmetro renal transversal; RIML: diâmetro renal longitudinal; ESC: comprimento da escápula; MEC: comprimento do metacarpo; COX: comprimento da pelve.

As variáveis $\mathrm{FC}, \mathrm{DBP}, \mathrm{CF}, \mathrm{CU}, \mathrm{DO}$, DT e DAT foram submetidas à análise de regressão linear múltipla. A seleção dos modelos realizada pelo método "backward", ao nível de $15 \%$ de probabilidade $(\mathrm{P}<0,15)$, permitiu estimar um modelo reduzido utilizando-se as variáveis fetométricas DBP, CF, CU, DO e DAT, o qual é representado pela seguinte equação: idade fetal $=$ $34,5+4,8 \mathrm{DBP}+9,9 \mathrm{CF}-7,5 \mathrm{CU}-2,3 \mathrm{DO}+$ $6,1 \mathrm{DAT}$

\section{DISCUSSÃO}

A utilização da ultra-sonografia em modo-B permitiu o acompanhamento das diversas modificações que ocorrem durante $\mathrm{O}$ desenvolvimento do concepto. A eficiência e a precisão dos métodos fetométricos $\mathrm{e}$ morfológicos utilizados neste estudo fortaleceram a explicação para tais modificações, concordando com os estudos realizados por Meira (1995). Cada exame teve duração de 30 a 50 minutos, coincidindo com o tempo dependido em trabalhos anteriores (Bailão, 1998; Léga, 2000), sendo que o número de observações realizadas por período foi maior ou igual a cinco para os parâmetros considerados de maior observação, e menor ou igual a quatro para os considerados de menor observação.

Nas fêmeas com gestação gemelar, mesmo que as variáveis aferidas não tenham coincidido para os dois indivíduos, os dados obtidos foram analisados conforme as médias dos mesmos, sendo este aspecto não considerado fator negativo para os resultados, pois, segundo Haibel (1988) e González de Bulnes et al. (1998), o número de fetos não influencia a eficiência da fetometria na prevenção da idade fetal.

Embora alguns autores considerem a estimativa da idade fetal mera especulação (Trigo, 2002), certamente o diagnóstico gestacional precoce, associado à determinação do número de fetos e à estimativa da idade fetal (Ishwar, 1995; Simplício, 2000; Salles, 2001), permite o aumento da produtividade do rebanho.

Apesar de muitos autores terem determinado equações fetométricas (Kähn et al., 1992; Bretzlaff et al., 1993; González de Bulnes et al., 
1998), vale salientar que cada equação se ajusta às condições nas quais o estudo foi realizado.

Neste estudo, o DBP teve alto coeficiente de determinação $\left(\mathrm{R}^{2}=0,93 ; \mathrm{P}<0,01\right)$, semelhante aos valores encontrados por Messias et al. (2001) e Souza et al. (2002), respectivamente iguais a 0,94 e 0,96 e diferente daquele encontrado por Chavez Moreno et al. (1996), igual a 0,74.

O DT foi aferido em secção horizontal, sugerida por Chavez Moreno et al. (1996), tendo apresentado alta correlação com a idade fetal $\left(\mathrm{R}^{2}=0,95 ; \mathrm{P}<0,01\right)$, sendo mais preciso que o índice encontrado por Aiumlamai et al. (1992), que foi $\mathrm{R}^{2}=0,80$.

Dois diâmetros abdominais foram relacionados com a idade fetal, DAT e DAP, sendo que ambas as regressões tiveram o mesmo coeficiente de determinação $\left(\mathrm{R}^{2}=0,91 ; \mathrm{P}<0,01\right)$. Souza et al. (2002) obtiveram resultados semelhantes que confirmam que essas medidas oferecem meio seguro para determinação do estádio gestacional.

Foram aferidos CF, CU, CR, CT, MET e MEC, sendo medidas somente as diáfises (Léga, 2000). Todas as regressões apresentaram coeficientes de determinação altos, variando de 0,93 a 0,96 $(\mathrm{P}<0,01)$, exceto a do MEC que teve coeficiente menor $\left(\mathrm{R}^{2}=0,87 ; \quad \mathrm{P}<0,01\right)$, mas ainda assim considerado acurado para se determinar a idade fetal.

Com relação às diáfises ósseas, o fêmur foi o osso mais correlacionado com a idade fetal em trabalhos anteriores, que mostraram coeficientes mais altos variando entre 0,82 e 0,89 (Souza et al., 2002); no entanto, esses valores foram menores do que os encontrados neste estudo. Não foram encontrados, na literatura consultada, estudos com CU, CT, CR, MEC, COX ou ESC que pudessem ser comparados a este trabalho.

O CU teve o maior coeficiente de determinação (Tab. 1) de idade fetal $\left(\mathrm{R}^{2}=0,96 ; \mathrm{P}<0,01\right)$, seguido pelo $\mathrm{CF}$ e DT $\left(\mathrm{R}^{2}=0,95 ; \mathrm{P}<0,01\right)$. Ainda com altos coeficientes de determinação, apareceram DBP, DOF e CR $\left(\mathrm{R}^{2}=0,93 ; \mathrm{P}<0,01\right)$. Dessa forma, pode-se afirmar que qualquer um desses parâmetros pode ser mensurado individualmente para determinar a idade fetal, com alto grau de acurácia. DIO e RIMT tiveram os menores coeficientes de determinação $\left(\mathrm{R}^{2}=0,67\right.$ e $\mathrm{R}^{2}=0,76$, respectivamente; $\left.\mathrm{P}<0,01\right)$, e os demais parâmetros, ESC, COX, MEC, RIML, DO e DBO, tiveram coeficientes de determinação variando de 0,80 a 0,89 , com $\mathrm{P}<0,05$ para $\mathrm{COX}$ e $\mathrm{P}<0,01$ para os demais, sugerindo-se que sejam associados com outras variáveis para avaliação de idade fetal.

As variáveis com maiores números de medidas foram submetidas a uma análise de regressão linear múltipla, a fim de se estabelecer um modelo que pudesse correlacionar mais de uma variável com a idade gestacional numa mesma equação; para tanto, somente as variáveis DBP, $\mathrm{CF}, \mathrm{CU}, \mathrm{DO}$ e DAT foram selecionadas com nível de significância de $15 \%$ de probabilidade.

Os movimentos somáticos e cardíacos fetais são parâmetros importantes para avaliação da viabilidade fetal e estão relacionados no estudo do perfil biofísico fetal.

A freqüência cardíaca diminui à medida que se aproxima o momento do parto (Chavez Moreno et al., 1996), variando de 250 a 105 batimentos por minuto, porém esta variável sofre influência do sistema nervoso autônomo e, portanto, não deve ser considerada como parâmetro único de determinação da idade fetal, mas sim para determinação da viabilidade fetal e do aspecto fisiológico da função cardíaca.

Em um estudo morfológico minucioso, várias medidas devem ser aferidas para se determinar com maior grau de precisão a idade fetal, e, sempre que possível, a relação entre as medidas deve ser considerada para aumentar o grau de acurácia. Porém, deve-se salientar que nem sempre todas as medidas de uma equação múltipla são passíveis de aferimento numa mesma oportunidade, visto que a posição e a movimentação do feto, a posição e a movimentação da mãe, o estádio gestacional, o tipo de equipamento e a experiência e paciência do operador podem influenciar os resultados. Portanto, a ultra-sonografia se configura como método eficiente para se atingir com sucesso os objetivos propostos neste trabalho e quanto maior for a sua demanda, certamente, tornar-se-á cada vez menos onerosa. 


\section{REFERÊNCIAS BIBLIOGRÁFICAS}

AIUMLAMAI, S.; FREDRICKSSON, G.; NISLFORS, L. Real-time ultrasonography for determinatin the gestacional ages of ewes. Vet. Rec., v.131, p.560-562, 1992.

AL-MERESTANI, M.R.; ZARKAWI, M.; WARDEH, M.F. Improving the reproductive efficiency, pregnancy diagnosis and monitoring the resumption of luteal activity in indigenous Damascus goats. Reprod. Domest. Anim., v.38, p.36-40, 2003

BAILÃO, L.A. Bases físicas da ultra-sonografia. Ribeirão Preto: Diagnosis - Curso de Aperfeiçoamento, 1998. p.1-37.

BRETZLAFF, K.N.; EDWARDS, J.; FORREST, D. et al. Ultrasonographic determination of pregnancy in small ruminants. Vet. Med., v.88, p.12-24, 1993.

CHALHOUB, M.; PRESTES, N.C.; LOPES, M.D. et al. Relación entre medidas fetales y uterina obtenidas por ecografia y edad de gestación em ovejas. In: CONGRESSO PANAMERICANO DE CIENCIAS VETERINARIAS, 16., 1998, Santa Cruz. Anales... Santa Cruz: Colegio de Medicos Veterinarios de Bolivia, 1998c. p.315.

CHAVEZ MORENO, J.; CHAVEZ STEINMANN, C.; BICKHARDT, K. Fetale herzfrequenzmessung und sonografhische Fetometrie zur Bestimmung des Trächtigkeitsstadiums bei Schaf. Dtsch. Tierarztl. Wochenschr., v.103, p.478-80, 1996.

DIGGLE, P.J.; LIANG, K.Y.; ZAGER, S.L. Analysis of longitudinal data. Oxford: Science Publication, 1995. $253 \mathrm{p}$.

GONZÁLEZ DE BULNES, A.; MORENO, J.S.; SEBASTIAN, A.L. Estimation of fetal development in Manchega dairy ewes by transretal ultrasonographic measurements. Small Rum. Res., v.27, p.243-250, 1998.

HAIBEL, G.K. Real-time ultrasonic fetal head measurement and gestational age in dairy goats. Theriogenology, v.30, p.1053-1057, 1988.

ISHWAR, A.K. Pregnancy diagnosis in sheep and goats: a review. Small Rum. Res., v.17, p.37-44, 1995.

KÄHN, W.; KÄHN, B.; RICHTER, A. et al. Ultrasonic examination of pregnant ewes. I -fetal measurement to estimate the stage of gestation and the probable date of lambing. Dtsch. Terarztl. Wochenschr., v.99, p.449-52, 1992.

KEEFER, C.L.; KEYSTON, R.; LAZARIS, A. et al. Production of clone goats after nuclear transfer using adult somatic cells. Biol. Reprod., v.66, p.199-203, 2002.

KUSINA, N.T.; CHINUWO, T.; HAMUDIKUWANDA, $\mathrm{H}$. et al. Effect of diferent dietary energy level intakes on efficiency of estrus synchronization and fertility in Mashona goat does. Small Rum. Res., v.39, p.283-288, 2001.
LÉGA, E. Acompanhamento ultra-sonográfico transabdominal em modo- $B$ e concentração sérica de progesterona dos primeiros 60 dias de gestação na cabra doméstica (Capra hircus, Linnaeus, 1758). 2000. 95f. Dissertação (Mestrado). - Faculdade de Medicina Veterinária e Zootecnia, Universidade Estadual Paulista, Jaboticabal, SP.

MEIRA, C. Aspectos fisiológicos e ultra-sonográficos durante o ciclo estral e a gestação de jumentas (Equus asinus). 1995. 88f. Tese (Doutorado) - Faculdade de Medicina Veterinária e Zootecnia, Universidade Estadual Paulista, Botucatu, SP.

MESSIAS, J.B.; OLIVEIRA, I.M.; OLIVEIRA, C.K. et al. Avaliação da ultra-sonografia em tempo real como método para predizer o peso fetal em caprinos. Rev. Bras. Reprod. Anim., v.25, p.151-152, 2001.

MONTGOMERY, D.C.; PECK, E. A. Introduction to linear regression analisys. New York: John Wiley \& Sons, 1982. 504p.

PANTER, K.E.; WEINZWEIG, J.; GARDNER, D.R. et al. Comparison of cleft palate induction by Nicotina glauca in goats and sheep. Teratology, v.61, p.203-210, 2000.

PREEZ, E.R.; DONKIN, E.F.; BOYAZOGLU, P.A. et al. Out of season breeding of milk goats - the effect of light treatment, melatonin and breed. J. S. Afr. Vet. Assoc., v.72, p.228-231, 2001

REICHLE, J.K.; HAIBEL, G.K. Ultrasonic biparietal diameter of second trimester Pygmy goat fetuses. Theriogenology, v.35, p.689-694, 1991.

SALLES, H.O. Um tratamento diferenciado para fêmeas prenhes. [S.I.]: Caprinet - O portal da Caprinoovinocultura, 2001. Disponível em: $<$ http://www.caprinet.com.br/artigo06012003-02.shtml $>$. Acessado em: 30 jun. 2003.

SAUERBREI, E.E.; NGUYEN, K.T.; NOLAN, R.L. Ultra-sonografia em ginecologia e obstetrícia (um guia prático). Porto Alegre: Artes Médicas, 1992. p.183-192.

SIMPLÍCIO, J.B. Manejo reprodutivo. [S.I.]: Caprinet O portal da Caprino-ovinocultura, 2000. Disponível em:<http://www.caprinet.com.br/tecnica3011200002.shtml $>$. Acessado em: 30 jun. 2003.

SOUZA, D.M.B.; WISCHRAL, A.; FARIAS, E. et al. Avaliação ultra-sonográfica do crescimento fetal em caprinos. Rev. Bras. Reprod. Anim., v.26, p.31-36, 2002.

TRIGO, A. Ecografias em ovinos. [S.I.]: Escola \& Comunidade, 2002. Disponível em: $<$ http://www.ipb.p/old/esa/EscolaCom/Circuitos/ECO/Ec ografias.html>. Acessado em: 10 jul. 2003.

USER'S guide: statistics. Version 6.03. Cary, NC: SAS Institute, 1996. 530p.

ZOU, X.G.; WANG, Y.G.; CHENG, Y. et al. M. Generation of clane goats (Capra hircus) from transfected foetal fibroblast cells, the effect of donor cell cycle. Mol. Reprod. Dev., v.61, p.164-172, 2002. 